\title{
A CHARACTERIZATION OF SIMPLY CONNECTED CLOSED ARCWISE CONVEX SETS
}

\author{
F. A. VALENTINE
}

Let $S$ be a set of points in the Euclidean plane $E_{2}$. It is our purpose to establish a necessary and sufficient condition that a simply connected ${ }^{1}$ closed set $S$ be arcwise convex. In order to do this precisely, the following notations and definitions are used.

Notation. The line determined by two distinct points $x$ and $y$ in $E_{2}$ is denoted by $L(x, y)$. We designate the open line segment joining $x$ and $y$ by $x y$, and the corresponding closed segment by $[x y]$. The two closed half-planes having $L(x, y)$ as a common boundary are designated by $R_{1}(x, y)$ and $R_{2}(x, y)$. The boundary of a set $K$ is represented by $B(K)$, and $H(K)$ denotes the convex hull of $K$. The complement of $S$ is denoted by $C(S)$.

Definition 1. A set $S \subset E_{2}$ is said to be unilaterally connected if, for each pair of distinct points $x$ and $y$ in $S$, there exists a continuum ${ }^{2}$ $S_{1} \subset S$ which contains $x$ and $y$, and which lies in one of the closed halfplanes determined by $L(x, y)$.

Definition 2. A set $S \subset E_{2}$ is said to be arcwise convex if each pair of points in $S$ can be joined by a convex arc lying in $S$. (A convex arc is one which is contained in the boundary of its convex hull.)

In a previous paper $[1]^{3}$ the author studied the complements of both arcwise convex sets and unilaterally connected sets. The theorem below establishes another intimate connection between these two concepts.

I am indebted to the referee for the following lemma which simplifies the proof of the theorem.

LEMMA. In order that a simply connected, connected, closed set $S \subset E_{2}$ be unilaterally connected, it is necessary that for each line $L$, all of the bounded components of $C(S)-L \cdot C(S)$ lie on the same side of $L$.

Proof. Suppose $L$ is a straight line for which a bounded component $D$ of $C(S)-L \cdot C(S)$ exists. Let $[x y]$ be the minimal closed interval containing $L \cdot B(D)$. Let $T$ be a continuum in $S$ which con-

Presented to the Society, November 25, 1950; received by the editors March 31, 1950 and, in revised form, October 1, 1950.

${ }^{1}$ A set $S C E_{2}$ is simply connected if each component of its complement is unbounded.

2 A continuum in $E_{2}$ is a bounded, closed, connected set.

${ }^{8}$ Number in brackets refers to the reference at the end of the paper. 
tains $x+y$, and which lies in a closed half-plane, denoted by $R_{1}(x, y)$, determined by $L$. There exists a circular circumference $Q$ which encloses $T+[x y]+B(D)$. Since $x+y \subset T$, no two arcs which intersect $Q$ but not $T$ can abut $[x y]$ from opposite sides. Let $A$ be an $\operatorname{arc}$ in $C(S)$ irreducible from $[x y]$ to $Q$. (By definition, $A$ contains no proper subarc containing points of $[x y]$ and points of $Q$.) Then $A$ abuts on $[x y]$, and it also contains an arc in $R_{2}(x, y)$ abutting on $[x y]$. Moreover, it is clear that $A \cdot D=0$.

Suppose that $D \subset R_{2}(x, y)$. Let $Q_{x}$ and $Q_{y}$ denote closed circular disks centered on $x$ and $y$ respectively, such that $\left(Q_{x}+Q_{y}\right) \cdot(A+Q)$ $=0$. There exists an arc $E \subset D+B\left(Q_{x}\right)+B\left(Q_{y}\right)$, having only its end points, $w$ and $z$, in $L \cdot x y$, such that $A \cdot x y$ is between $w$ and $z$ on $L$. Then $E+w z$ is a simple closed curve enclosed by $Q$ and lying in $R_{2}(x, y)$. Since $A$ abuts on $[x y]$ via $R_{2}(x, y)$, the above implies that $A-A \cdot x y$ lies within the region bounded by $E+w z$. This is a contradiction, so that we have $D \subset R_{1}(x, y)$.

If $U$ is any other bounded component of $C(S)-L \cdot C(S)$, let $[p q]$ denote the minimal closed interval of $L$ containing $L \cdot B(U)$. Each pair of the four points $x, y, p, q$ (whether distinct or not) is contained in a continuum in $S$ lying in $R_{1}(x, y)$ or in $R_{2}(x, y)$. From this fact it follows readily that there exists a continuum $T^{\prime} \subset S$ which contains $x+y+p+q$, and which lies in $R_{1}(x, y)$ or in $R_{2}(x, y)$. From the above paragraph we must have $T^{\prime} \subset R_{1}(x, y)$ since $D \subset R_{1}(x, y)$. Hence, we must also have $U \subset R_{1}(x, y)$. This completes the proof.

Theorem. A necessary and sufficient condition that a simply connected closed set $S \subset E_{2}$ be arcwise convex is that it be unilaterally connected.

Proof. It is the sufficiency which requires proof, since the necessity is obvious. Choose $x \in S, y \in S$. If $x y \subset S$, then $x$ and $y$ can be joined by a convex arc in $S$. Hence, suppose $x y \nsubseteq S$. By hypothesis, there exists a continuum $S_{1} \subset S$ containing $x$ and $y$ and lying in $R_{1}(x, y)$ or in $R_{2}(x, y)$. Suppose $S_{1} \subset R_{1}(x, y)$. Choose any point $\alpha \in x y \cdot C(S)$. Define $K(\alpha)$ to be that component of $C(S) \cdot R_{1}(x, y)$ which contains $\alpha$. Since $S_{1}$ is a bounded closed connected set in $R_{1}(x, y)$, and since we can establish an order $x<\alpha<y$ on $L(x, y)$, we have $K(\alpha) \subset H\left(S_{1}\right)$. Hence the set sum $\sum K(\alpha)$ ( $\alpha$ ranges over $C(S) \cdot x y$ ) is bounded. Define the set sum $T$ to be

$$
T \equiv x+y+\overline{\sum K(\alpha)} \quad(\alpha \text { ranges over } C(S) \cdot x y) .
$$

It follows with the help of the preceding lemma that $C \equiv B(H(T))-x y$ is a convex arc lying in $S$. This proves the theorem. 
The above characterization does not apply to sets which are not simply connected. For instance, the set $S$ consisting of the circumference of a circle $C$ plus a single outward normal to $C$ (segment or halfline) is unilaterally connected but not arcwise convex. A nontrivial characterization of non-simply connected arcwise convex sets appears to be difficult to determine.

\section{REFERENCE}

1. F. A. Valentine, Arcwise convex sets, Proceedings of the American Mathematical Society vol. 2 (1951) pp. 159-165.

University of California, Los Angeles 